\title{
利用通道注意力与分层残差网络的图像修复
}

\author{
杨昊，余映 ${ }^{*}$ \\ (云南大学信息学院 昆明 650091) \\ (yuying.mail@163.com)
}

\begin{abstract}
摘 要: 针对现有深度学习图像修复方法对不同尺度特征的感知和表达能力存在不足的问题, 提出一种利用多尺度 通道注意力与分层残差网络的图像修复模型. 首先采用 U-Net 作为生成器的主干网络, 实现对破损图像的编码与解 码操作; 然后通过在编码器与解码器中分别构建多尺度的分层残差结构, 以增强网络提取和表达破损图像特征的能 力; 最后在编码器与解码器间的跳跃连接中嵌人扩张的多尺度通道注意力模块, 以提高模型对编码器中图像低级特 征的利用效率. 实验结果表明, 在人脸、街景等数据集的破损图像修复上, 该模型在主观视觉感受和客观评价指标方 面均优于其他经典的图像修复方法.
\end{abstract}

关键词: 图像修复; 深度学习; 多尺度; 通道注意力

中图法分类号: TP391.41 DOI: 10.3724/SP.J.1089.2021.18514

\section{Image Inpainting Using Channel Attention and Hierarchical Residual Networks}

\author{
Yang Hao and Yu Ying* \\ (School of Information Science and Engineering, Yunnan University, Kunming 650091)
}

\begin{abstract}
Existing deep-learning-based inpainting methods may have some shortcomings in perceiving and presenting image information at multi-scales. For this problem, we proposed an image inpainting model based on multi-scale channel attention and a hierarchical residual backbone network. Firstly, we adopted a U-Net architecture as the generator backbone of our inpainting model to encode and decode the damaged image. Secondly, we built multi-scale hierarchical residual structures in the encoder and decoder respectively, which can improve the ability of the model to extract and express occluded image features. Finally, we designed a dilated multi-scale channel-attention block and inserted it into the skip-connection of the generator. This block can improve the utilization efficiency of low-level features in the encoder. Experimental results show that our model outperforms other classical inpainting approaches in the face, street-view inpainting tasks, both qualitatively and quantitatively.
\end{abstract}

Key words: image inpainting; deep learning; multi scale; channel attention

图像修复利用破损图像或训练图像数据集中 的先验信息为缺失或损坏的图像区域合成视觉逼 真且语义正确的替代内容. 近年来其被广泛地应 用于生物医学、电影特效和受损文物复原等诸多专
业技术领域, 目前已成为智能图像处理、计算机视 觉和机器学习等方面的研究热点.

传统的图像修复方法主要分为基于扩散的方 法 $^{[1]}$ 和基于样本块的方法 ${ }^{[2]}$. 基于扩散的方法通过

收稿日期：2020-07-24; 修回日期：2021-01-29. 基金项目：国家自然科学基金(61263048); 云南省应用基础研究计划(2018FB102); 云南大学 “中青年骨干教师培养计划” XT412003). 杨昊(1995一), 男, 硕士研究生, 主要研究方向为图像处理、计算机视觉、深度学 习; 余映(1977一), 男, 博士, 副教授, 硕士生导师, 论文通讯作者, 主要研究方向为图像与视觉、人工神经网络. 
求解偏微分方程将已知区域的图像内容平滑地传 递到缺失的图像区域; 该类方法仅能修复较小的 受损图像区域. 基于样本块的方法通过在已知的 图像区域内搜寻匹配较好的替换块, 将其逐个复 制到相应的缺失区域来修复受损的图像; 该类方 法可以修复较大的图像缺失区域, 但与基于扩散 的方法一样无法捕获受损图像中的高级语义, 更 无法真实地生成复杂且非重复的图像结构和纹理.

近年来, 基于深度学习的图像修复方法取得 了许多突破性的进展. 通过使用大规模的数据集 训练图像修复网络, 使其能够捕获受损图像中的 高级语义特征, 从而生成高质量的图像修复结果. 生成器是修复模型中最重要的组成部分, 而生成 器的主干网络广泛采用的是 U-Net 结构 ${ }^{[3]}$. 原始的 U-Net 不能完全满足图像修复任务的需求, 因此需 要在网络结构上进行改进. 为了提高对受损图像 中有效像素的利用率, Liu等 ${ }^{[4]}$ 将 U-Net 结构中的普 通卷积替换成部分卷积(partial convolution, PConv). Yan 等 ${ }^{[5]}$ 在 U-Net 的解码器中添加了一个特殊的移 位连接层, 有效地结合基于样本块的方法与基于 卷积神经网络(convolutional neural networks, CNN) 的方法. Liu 等 ${ }^{[6]}$ 为了获得更准确的修复结果, 使 用 2 个结构不同的 U-Net 分别对受损图像进行粗略 修复和精细完善. 尽管上述基于 U-Net 的图像修复 模型取得了较好的结果, 但它们都不能有效地利 用 U-Net 结构中的跳跃连接来捕获受损图像在编 码器中的低级特征.

对于大尺度的破损图像修复任务, 捕获相隔 较远图像区域间的特征相关信息非常重要. 例如, 在人脸图像修复任务中, 当人脸被矩形掩码遮盖 后，五官几乎完全不可见．在此情况下，图像修 复网络需要很好地捕获相隔较远的图像特征间的 相关信息，才能有效地表达图像中不同尺度的特 征, 从而生成真实且清晰的面部结构. 为了提高 修复模型对相隔较远图像区域间的特征信息相关 性的捕获能力, $\mathrm{Yu}$ 等 $^{[7]}$ 设计了一个上下文注意力 网络层, 对受损图像特征的相关性进行建模, 但 其需要构建一个精炼网络来对图像修复模型进行 端到端的训练. Ren 等 ${ }^{[8]}$ 提出了一种利用外观流的 修复模型, 通过采样具有相似结构的图像内容来 引导图像修复的过程; 但为了得到修复图像的外 观流, 其需要引人额外的外观流的预测模块和采 样损失函数.

针对目前深度修复方法对不同尺度图像特征 的感知和表达存在的不足, 本文提出一种称为
Res2U-Net 的生成器主干网络, 可以适用于各种场 景图像修复任务. 首先, 针对 Res2U-Net 的编码器 和解码器分别构建了相应的分层残差结构, 该结 构能够显著地提升图像修复的质量. 其次, 为高效 地利用编码器网络中的低级特征, 设计了一种扩 张的多尺度通道注意力 (dilated multi-scale channel-attention, DMSCA)模块, 将其嵌人到生成器网 络的跳跃连接中. DMSCA 模块能够根据各类图像 特征的重要程度, 对各个特征通道进行多尺度自 适应加权. 感受野的扩大能够更好地捕获受损图 像中相隔较远特征间的信息相关性, 特征尺度的 增加能够有效地提高提取与表达受损图像多尺度 特征的能力.

\section{1 相关工作}

\section{1 基于深度学习的图像修复}

基于深度学习的图像修复方法将受损图像编 码为高级特征, 以此来引导图像修复模型生成缺 失的图像内容. Pathak 等 ${ }^{[9]}$ 提出了上下文编码器 (context encoder, CE), 最早在图像修复模型中采 用编码-解码的 CNN 结构, 并利用重构损失与对抗 损失来共同改善修复模型的训练过程, 但 CE 模型 仅能修复低分辨率的破损图像. 在 CE 模型的基础 上, Iizuka 等 ${ }^{[10]}$ 利用全局鉴别器和局部鉴别器来联 合训练图像修复模型，对于规则的图像破损区域, 其修复图像的全局结构连贯、局部细节逼真; 但对 不规则破损图像区域, 该模型修复效果有限. 为了 提高图像修复模型对不规则受损图像区域的修复 能力, Liu 等 $^{[4]}$ 提出了一种仅依赖于受损图像中有 效像素的 PConv. 此后, Yu 等 ${ }^{[11]}$ 设计了一种能够自 动更新不规则掩码的门控卷积, 进一步改善了图 像修复模型对不规则破损的修复效果. 为了使修 复结果具有更准确的细节, Edge-Connect 模型 ${ }^{[12]}$ 通 过预测受损图像的边缘信息来辅助图像修复过程, 降低修复的难度; 但该模型需要设计额外的辅助 网络, 增加了训练与测试的成本.

\section{2 注意力机制}

在深度学习中, 注意力机制能够根据输人特 征的重要程度来为模型合理地分配计算资源. 按 照注意力机制应用的维度, 可分为空间注意 ${ }^{[13]}$ (spatial attention, SA)与通道注意力 ${ }^{[14]}$ (channel attention, CA). 由于空间维度的上下文特征在图像 修复过程中可以作为受损图像区域的参考对象, 文献[6-7,15-16]都将 SA 机制应用于图像修复网络 
中. $\mathrm{Yu}$ 等 ${ }^{[7]}$ 提出了一种上下文注意力层，该结构利 用 SA 为缺失图像区域找到与其最相似的背景内 容. Wang 等 ${ }^{[15]}$ 在上下文注意力层的基础上, 提出 了一种多尺度的图像上下文注意力的学习策略, 使修复模型能够更灵活地处理丰富的背景信息. 在此之后, Liu 等 ${ }^{[6]}$ 提出了一种连贯语义注意力层, 通过 SA 来提升修复的受损图像区域内部的空间语 义一致性. 为使图像修复结果在视觉和语义上保持 一致, Zeng 等 ${ }^{[16]}$ 提出了一种金字塔上下文编码器网 络(pyramid context encoder networks, PEN-Net), 利 用金字塔网络结构将从高级语义特征中学到的注 意力信息传递到低级特征中.

\subsection{U-Net 结构}

U-Net 是 Ronneberger 等 ${ }^{[3]}$ 提出的一种基于编 码-解码架构的 CNN, 最初应用于医学图像处理领 域中的细胞图像分割任务. 由于 U-Net 的跳跃连接 操作能够将编码器中的低级特征与解码器中的深 层语义特征相结合, 因此其在深度图像修复任务 中具有很好的应用潜力. 为使修复模型能够更好 地利用受损图像中的有效像素, Liu 等 ${ }^{[4]}$ 提出一种 利用掩码对输人特征加权的 PConv, 以此替换 U-Net 中的普通卷积. Xie 等 ${ }^{[17]}$ 利用可学习双向注 意力模块改善 PConv 的掩码更新过程, 提出一种 基于 14 层 U-Net 的修复模型. Liu 等 ${ }^{[6]}$ 采用 2 个不
同结构的 U-Net 来实现对图像的分阶段修复，其在 第 2 个 U-Net 中引人连贯语义注意力来提升图像修 复质量. 为了使修复的人脸图像具有更精细的面 部纹理, Fang 等 ${ }^{[18]}$ 设计了一种混合空洞卷积, 将它 和谱归一化模块相结合, 并添加到 U-Net 当中.

\section{2 本文模型}

为了使图像修复结果具有更准确的语义内容 与更精细的纹理, 本文提出了一种基于多尺度 CA 与分层残差主干网络的图像修复模型(multi-scale channel attention and hierarchical residual backbone networks, MS-CAHRBN). MS-CAHRBN 的整体结 构如图 1 所示, 其组成部分包括 Res2U-Net, DMSCA 模块, 移位连接层和鉴别器. 分层残差结 构能够有效地扩大 Res2U-Net 的感受野，同时还能 增加输出特征尺度的数量, 明显地提升图像修复 的质量. DMSCA 模块位于生成器最外 2 层的跳跃 连接中, 它能使解码器更有效地利用对应编码器 中的低级特征. 移位连接层来自 Shift-Net 修复模 型 ${ }^{[5]}$, 它利用 SA 获取已知区域图像的特征来改善 图像修复质量. 鉴别器用于判别接收到图像的真 假, 本文采用马尔可夫判别器 ${ }^{[19]}$. 下面将具体阐 述本文的 Res2U-Net 和 DMSCA 模块.

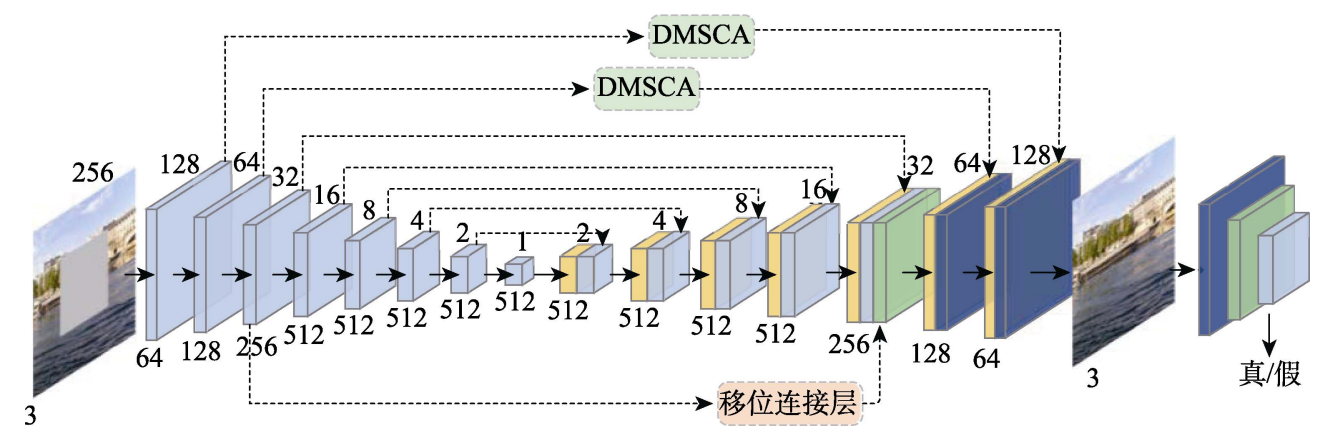

图 1 MS-CAHRBN 网络的整体结构

\subsection{Res2U-Net}

为了让生成模型更好地捕获相隔较远图像区 域间的特征相关信息，一种简单的方法是扩大生 成模型的感受野大小，从而获取更多的全局信息. 在目标检测中, Gao 等 ${ }^{[20]}$ 提出了一种称为 Res $2 \mathrm{Net}$ 的残差网络，利用分层残差结构扩大检测模型的 感受野范围，提升其对多尺度目标的检测效率。受 此启发, 本文通过在编码器与解码器中分别构造 分层残差结构来增大 U-Net 的感受野的范围, 并将 改造后的 U-Net 称为 Res2U-Net. 采用 Res2U-Net 作为生成器主干，不仅能提高修复模型对相隔较
远图像区域间的特征信息相关性的捕获能力，还 能增强模型对多尺度特征的提取与表达能力. 图 2 所示为其基本构成.

在 Res2U-Net 中, 编码器和解码器分别被称为 Res2-encoder 和 Res2-decoder. 其中, Res2-encoder 是 Res2U-Net 的下采样部分, 用于提取受损图像的 多尺度特征, 其基础模块如图 2a 所示. 在下采样 过程中, 首先将输人特征 $F_{\text {in }}$ 送人 $1 \times 1$ 的普通卷积 来调整其通道权重, 为了增加编码器中不同尺度 的感受野数量, 将加权后的输人特征以通道维度 平均分成 4 组, 并送人分层残差结构中进行, 该处 


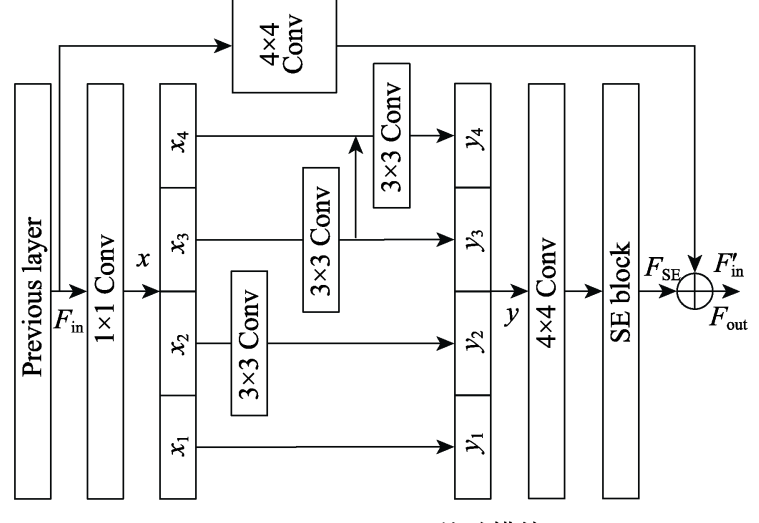

a. Res2-encoder 基础模块

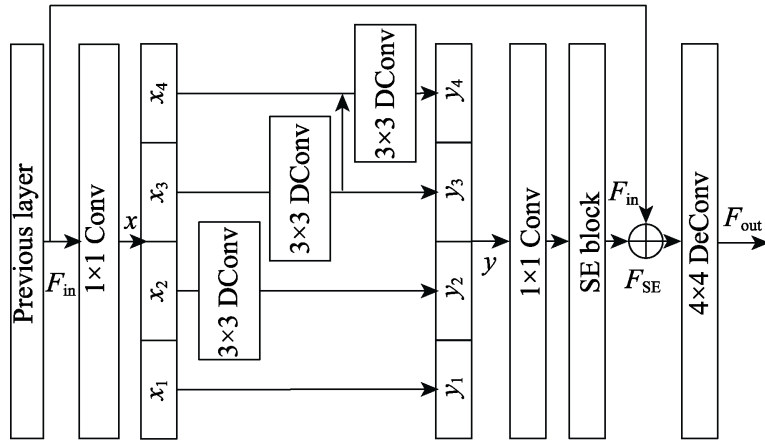

b. Res2-decoder 基础模块

图 2 Res2U-Net 的构成

理过程可表示为

$$
y_{i}=\left\{\begin{array}{ll}
x_{i}, & \text { if } i=1 \\
W_{i} * x_{i}, & \text { if } i=2 \\
W_{i} *\left(y_{i-1}+x_{i}\right), & \text { otherwise }
\end{array} .\right.
$$

其中, $x_{i}(i=1,2,3,4)$ 为分组后的输人特征; $W_{i}(i=2,3,4)$ 为与其对应的分组输人特征的 $3 \times 3$ 卷 积的权重; $y_{i}(i=1,2,3,4)$ 为每组操作的输出特征. 除第 1 组外, 每组操作都将上一组卷积的计算结果 加人该组的输人特征中, 此操作逐步增大 $y_{i}(i=1,2,3,4)$ 的感受野.

为了进一步融合各个尺度的特征，将分层残 差结构的输出特征 $y_{i}(i=1,2,3,4)$ 沿着通道维度组 合为 $y=\operatorname{Cat}\left(y_{1}, y_{2}, y_{3}, y_{4}\right)$. 其中, $\operatorname{Cat}(\cdot)$ 表示对特征 进行跨通道的级联操作.

容易看出，经过上述分层残差结构处理以后，
特征 $y$ 中包含了多种不同尺度的感受野, 这有利 于 Res2-encoder 融合输人特征中不同尺度的空间 信息，提高修复模型提取受损图像多尺度特征的 能力. $y$ 中大尺度的感受野也有助于修复模型捕 获受损图像中相隔较远特征间的信息相关性.

为使 Res2-encoder 在编码过程中保留更多的有 效信息，本文没有采用原始 U-Net 中的池化层，而 是通过一个步长为 2 的 $4 \times 4$ 卷积来对特征 $y$ 进行下 采样, 并通过挤压激励(squeeze-and-excitation, SE) 模块 ${ }^{[21]}$ 对采样结果进行通道维度的自适应调整, 调整后的特征记为 $F_{\mathrm{SE}}$.

除上述处理外, 本文还对输人特征 $F_{\mathrm{in}}$ 利用另 一个 $4 \times 4$ 卷积进行下采样, 采样结果记为 $F_{\text {in }}^{\prime}$, 其 尺度与 $F_{\mathrm{SE}}$ 相同. 最后, 本文将 $F_{\mathrm{in}}^{\prime}$ 以残差的方式 与 $F_{\mathrm{SE}}$ 相加融合, 获得 Res2-encoder 基础模块的最 终输出结果 $F_{\mathrm{out}}=F_{\mathrm{SE}}+F_{\mathrm{in}}^{\prime}$. 这种残差融合的方式 有助于改善生成模型在训练时梯度消失或爆炸的 问题.

Res2-decoder 是 Res2U-Net 的上采样部分, 用 于生成修复图像中不同尺度的特征，其基础模块 如图 2b 所示. Res2-decoder 与 Res2-encoder 在结构 上大体相似，二者的主要区别在于，Res2-decoder 为了在上采样的过程中进一步扩大 Res2U-Net 的 感受野, 将其中分层结构的普通卷积替换为了填 充量为 2 的扩张卷积; 这有利于模型在修复缺失图 像内容时捕获更大范围的特征间相关信息. 除此 之外, 为了实现上采样, 将输出特征恢复到与输人 破损图像相同的尺度, 本文在 Res2-decoder 基础模 块的最外层中添加了一个反卷积. 上采样过程可 表示为 $F_{\text {out }}=\operatorname{DeConv}\left(F_{\mathrm{SE}}+F_{\text {in }}\right)$. 其中, $\operatorname{DeConv}(\cdot)$ 表示卷积核大小为 4 的反卷积函数.

\subsection{DMSCA 模块}

CA 机制的特点在于能够通过对特征间的相对 重要性建模来调整各通道的特征. 普通的 CA 模块 如图 3 所示. 为了更有效地利用编码器中的低级特 征, 本文提出一种利用扩张与多尺度操作的 DMSCA 模块，其结构如图 4 所示.

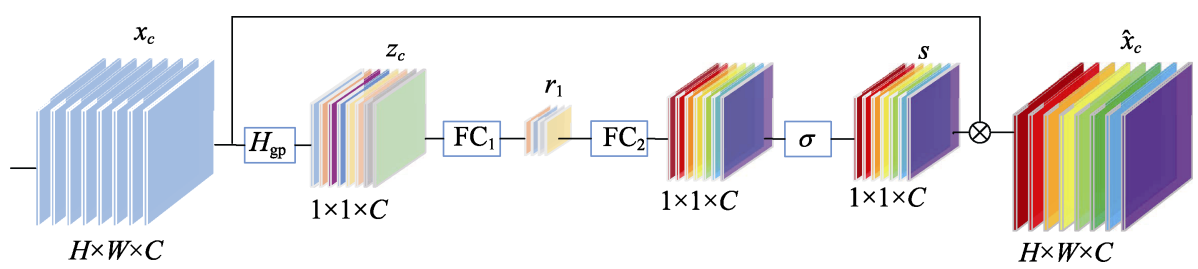

图 3 普通的 CA 模块 


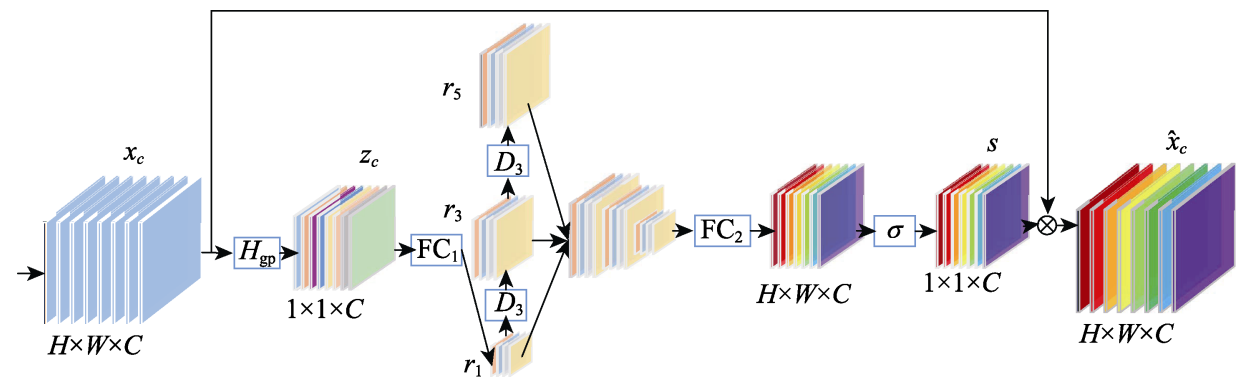

图 4 DMSCA 模块

编码器中输人的低级特征被记为 $x_{c} \in \mathbb{R}^{H \times W \times C}$, 其尺度为 $H \times W$, 通道数为 $C$. 在 DMSCA 模块中, 特征 $x_{c}$ 首先被送人全局平均池化层 $H_{\mathrm{gp}}(\cdot)$ 中, 以 提取各通道的全局空间特征 $z_{c} \in \mathbb{R}^{C}$, 该过程可表 示为

$$
z_{c}=H_{\mathrm{gp}}\left(x_{c}\right)=\frac{1}{H \times W} \sum_{i=1}^{H} \sum_{j=1}^{W} x_{c}(i, j) .
$$

其中, $x_{c}(i, j)$ 是 $x_{c}$ 位于第 $c$ 通道坐标为 $(i, j)$ 的特 征值. 对于普通的 CA 模型 ${ }^{[14]}$, 在得到全局空间特 征 $z_{c}$ 后，便利用门控机制来捕获特征通道之间的 相关性，其输出特征依赖关系为 $r_{1}=\delta\left(\mathrm{FC}_{1}\left(z_{c}\right)\right)$. 其中, $\delta(\cdot)$ 为 $\mathrm{ReLU}$ 激活函数; $\mathrm{FC}_{1}(\cdot)$ 为全连接层.

为了更好地处理不同尺度的图像特征，将 $r_{1}$ 连续通过 2 个相同的 $3 \times 3$ 扩张卷积(填充量为 2 , 步 长为 2), 以增大 DMSCA 模块的感受野范围. 正如 Ioffe 等 ${ }^{[22]}$ 指出的, 当 2 个 $3 \times 3$ 的卷积级联时, 其感 受野的大小相当于一个 $5 \times 5$ 的卷积, 该策略有助 于减少参数与计算时间. 扩张后的结果分别记为 $r_{3}=\delta\left(D_{3}\left(r_{1}\right)\right)$ 和 $r_{5}=\delta\left(D_{3}\left(r_{3}\right)\right)$. 其中， $D_{3}(\cdot)$ 表示扩 张卷积操作. 为了结合不同尺度的特征依赖关系, 对 $r_{1}, r_{3}$ 和 $r_{5}$ 进行跨通道的级联, 并将级联结果送 人上采样层 $\mathrm{FC}_{2}(\cdot)$ 中, 以恢复到与 $z_{c}$ 相同的尺度. 再利用 sigmoid 函数 $\sigma(\cdot)$ 获得各通道相应的多尺度 权重 $s=\sigma\left(\mathrm{FC}_{2}\left(\operatorname{Cat}\left(r_{1}, r_{3}, r_{5}\right)\right)\right)$. 最终, 将权重 $s$ 加权 到对应的未经处理的低级特征 $x_{c}$ 上, 得到校准后 的输出特征 $\hat{x}_{c}=s \cdot x_{C}$. 通过加权后的低级特征 $\hat{x}_{C}$, 解码器获取了低级特征中的重要内容.

\section{3 损失函数}

为了让图像修复结果的纹理细节更清晰, 语 义内容更合理，本文将多种损失函数结合起来作 为修复模型的联合训练损失, 定义为

$$
L=\lambda_{\text {rec }} L_{\text {rec }}+\lambda_{\text {cont }} L_{\text {cont }}+\lambda_{\text {sty }} L_{\text {sty }}+\lambda_{\text {tv }} L_{\text {tv }}+\lambda_{\text {adv }} L_{\text {adv }} \text {. }
$$

其中， $L_{\mathrm{rec}}$ 为像素重构损失; $L_{\mathrm{cont}}$ 为内容损失;
$L_{\mathrm{sty}}$ 为风格损失; $L_{\mathrm{tv}}$ 为总变分损失 ${ }^{[23]} ; L_{\mathrm{adv}}$ 为对抗 损失 ${ }^{[24]}$. $\lambda_{\text {rec }}, \lambda_{\text {cont }}, \lambda_{\mathrm{sty}}, \lambda_{\mathrm{tv}}$ 和 $\lambda_{\mathrm{adv}}$ 分别为各损失函 数的权重值. 下面将逐一介绍各损失函数的定义.

\subsection{1 像素重构损失}

$I^{\text {in }}$ 表示输人的损坏图像， $I^{\text {out }}$ 表示模型的修 复结果, $I^{\mathrm{gt}}$ 表示对应的真实图像. 像素重构损失 是通过采用 $L_{1}$ 损失函数来衡量 $I^{\text {out }}$ 与 $I^{\mathrm{gt}}$ 之间的像 素差异, 定义为 $L_{\mathrm{rec}}=\left\|I^{\mathrm{out}}-I^{\mathrm{gt}}\right\|_{1}$.

\subsection{2 内容损失}

内容损失并不是直接计算原图与修复结果在 图像空间的距离, 而是比较二者在已预训练好的 网络中高维特征的相似度, 其计算公式为

$$
L_{\text {cont }}=\sum_{l=1}^{N} \frac{1}{h_{l} w_{l} c_{l}}\left\|\boldsymbol{\Phi}^{l}\left(I^{\mathrm{gt}}\right)-\boldsymbol{\Phi}^{l}\left(I^{\mathrm{out}}\right)\right\|_{2} .
$$

其中, $\boldsymbol{\Phi}^{l}(I)$ 是从预训练的 VGG-16 网络 ${ }^{[25]}$ 的池化 层中提取的图像 $I$ 的第 $l$ 层特征图; $h_{l}, w_{l}$ 和 $c_{l}$ 分 别是 $\boldsymbol{\Phi}^{l}(I)$ 的长度、宽度以及通道数.

\subsection{3 风格损失}

为了确保修复后的图像 $I^{\text {out }}$ 具有与原始图像 $I^{\mathrm{gt}}$ 相同的图像风格，本文进一步引人风格损失 $L_{\text {sty }}=\sum_{l=1}^{N} \frac{1}{c_{l}^{2}}\left\|\boldsymbol{M}_{\mathrm{Gram}}\left(I^{\mathrm{out}}\right)-\boldsymbol{M}_{\mathrm{Gram}}\left(I^{\mathrm{gt}}\right)\right\|_{2}$. Gram 矩阵 是通过计算内积的 Hermitian 矩阵得到的, 通常用于 计算特征间的相关性. 而 $\boldsymbol{M} \operatorname{Gram}(I)=\boldsymbol{\Phi}^{l}(I)^{\mathrm{T}} \boldsymbol{\Phi}^{l}(I)$.

\subsection{4 总变分损失}

总变分损失能使图像修复结果更为平滑, 在 训练过程中降低噪声对修复结果的影响, 定义为

$$
L_{\mathrm{tv}}=\sum_{m, n}\left(\left(I_{m, n+1}-I_{m, n}\right)^{2}+\left(I_{m+1}-I_{m, n}\right)^{2}\right) .
$$

其中, $m$ 和 $n$ 表示 $I^{\text {out }}$ 中像素的位置坐标.

\subsection{5 对抗损失}

本文利用对抗损失来监督修复模型的训练过 程, 对于模型中的鉴别器 $D$, 其对抗损失函数被 定义为 


$$
\begin{gathered}
L_{\text {adv }}=\min _{\Theta} \max _{D} E_{I^{\mathrm{gt}} \sim p_{\text {data }}\left(I^{\mathrm{gt}}\right)}\left[\lg D\left(I^{\mathrm{gt}}\right)\right]+ \\
E_{I^{\text {out }} \sim p_{\text {miss }}\left(I^{\text {out }}\right)}\left[\lg \left(1-D\left(I^{\text {out }}\right)\right)\right] .
\end{gathered}
$$

其中, $p_{\text {data }}\left(I^{\mathrm{gt}}\right)$ 表示真实图像的分布; $p_{\text {miss }}\left(I^{\text {out }}\right)$ 表示修复图像的分布; $I^{\mathrm{gt}}$ 与 $I^{\text {out }}$ 在尽可能地使该 式的结果最小化; 而 $D$ 却在尽可能地使结果最大 化，修复模型在这种对抗中不断优化.

\section{3 实验结果与分析}

为了客观且全面地评价图像修复模型的性能, 本节通过定性评价、定量评价以及用户评价来全面 比较各种模型的图像修复结果. 此外, 还通过消融 实验与可视化实验来分析本文各模块对修复过程 的作用. 实验采用的公开数据集 CelebA- $\mathrm{HQ}^{[26]}$ 与 Paris StreetView ${ }^{[27]}$. CelebA-HQ 为人脸数据集, 包 含 30000 幅高清正面人脸图像, 其中 28000 幅作为 训练集, 2000 幅作为测试集. Paris StreetView 为街 景数据集, 其训练集为 14900 幅, 测试集为 100 幅. 对于 CelebA-HQ, 训练图像的大小直接调整为 $256 \times 256$ 像素. 对于 Paris StreetView, 首先将图像 的最短边等比例调整为 350 像素, 然后从中裁剪 $256 \times 256$ 像素的子图像进行训练. 训练过程中修 复模型使用 Adam 算法进行优化, 初始学习率设置 为 0.0002 , Batch size 设置为 1 . 损失函数各部分的 权重分别设置为 $\lambda_{\text {rec }}=1.0, \lambda_{\text {cont }}=1.0, \lambda_{\text {sty }}=10.0$, $\lambda_{\mathrm{tv}}=0.01$ 和 $\lambda_{\mathrm{adv}}=0.2$. 此外, 本文采用 Pytorch 框架 训练和测试模型, 实验平台配置为 Intel Core i7-6850K $3.60 \mathrm{GHz}$ CPU, NVIDIA GeForce GTX 1080Ti GPU

\section{1 定性评价}

矩形中心掩码是图像修复任务中最为常见的 图像破坏形式. 在定性评价中, 本文采用大小为 $128 \times 128$ 像素的正方形中心掩码. 图 5 所示为本文 模型与其他 4 种模型在修复破损图像的对比结果. 其中, 前 5 行为人脸图像, 后 5 行为街景图像; 对 比模型分别为 $\mathrm{GMCNN}^{[28]}$, PIC ${ }^{[29]}$, Shift-Net ${ }^{[5]}$ 和 PEN-NET ${ }^{[16]}$.

Shift-Net 使用移位连接层来使图像修复结果 看起来更加真实, 但在修复小尺度的图像细节时, 修复结果中容易出现模糊的图像区域. 例如, 在图 $5 \mathrm{c}$ 的第 1 张图片中，其修复的眼镜缺少细节; 第 8 张图片中的窗户与第 9 张图片中的拱门都出现了 局部的模糊. GMCNN 在表征修复图像的整体内容
方面表现良好, 但其修复结果有时会出现一些结 构和纹理上的失真, 可从图 $5 \mathrm{~d}$ 的第 1 张图片中的 嘴部细节与第 7 张图片中的铁窗顶部边缘看出; 在 第 9 张图片中, 拱门的修复结果甚至出现了重复的 伪影. PIC 采用一种概率策略来生成多元化的修复 结果, 从图 5e 可以看出, 其对于街景图像的修复 效果较好, 但当受损图像具有复杂的背景信息时, 其修复效果明显劣化. 从第 3 张图片可以看出, PIC 完全没有修复出眼镜结构, 第 4 张图片中人的 眼部特征略显怪异, 第 5 张图片中发色出现偏差. 在图 $5 \mathrm{f}$ 中, PEN-NET 对于眼部细节的修复较为精 细, 但其对于第 1 张与第 3 张图片中的眼镜完全没 能恢复，且修复区域与已知区域间有明显的分界 线; 在第 9 张图片中, 拱门的修复结果出现了伪 影. 从图 $5 \mathrm{~g}$ 可以看出, 本文模型对于面部特征的 修复十分精致, 缺失的眼镜也被完整地恢复出来, 这证明了其能较好地把握受损图中远距离特征间 的信息相关性. 对于街景图像的建筑结构, 本文模 型能较为准确地推断出来, 且门窗的纹理也未产 生大的畸变. 总之, 本文模型的修复结果在语义结 构和细节纹理 2 个方面均明显优于其他对比模型.

\section{2 定量评价}

为了客观评价本文模型的图像修复效果, 本 节选取上文中矩形中心破损修复的实验结果进行 定量比较. 峰值信噪比 (peak signal-to-noise ratio, PSNR)与结构相似度 (structural similarity, SSIM)是 衡量图像修复质量最常用的指标; PSNR 比较图像 在像素层面上的差别, SSIM 比较图像在亮度、对 比度与结构上的差别. 当图像受损区域较大时, 会 出现修复结果与原图存在一定差异, 但在感官上 合乎人类感知的情况. 此时, 仅凭 PSNR 与 SSIM 无法完全体现修复结果给人的主观感受. 因此, 本 文引人弗雷歇距离(Fréchet inception distance, FID) 作为评价指标, 通过计算真实图像集和生成图像 集在高级特征层面的距离来反映模型的图像生成 质量. PSNR 与 SSIM 的数值越大, 表示修复质量越 好; FID 的数值越小, 表示修复质量越好.

表 1 和表 2 分别为本文模型与对比模型在人脸 数据集和街景数据集上修复中心矩形破损的性能 指标, 各指标的最佳值用粗体表示. 从中可以看 出, 本文模型的 3 项评价指标 PSNR, SSIM 和 FID 均优于其他对比模型. 说明无论是在像素层面和 结构层面, 还是在感知层面, 本文模型的图像修复 质量均优于其他对比模型。 

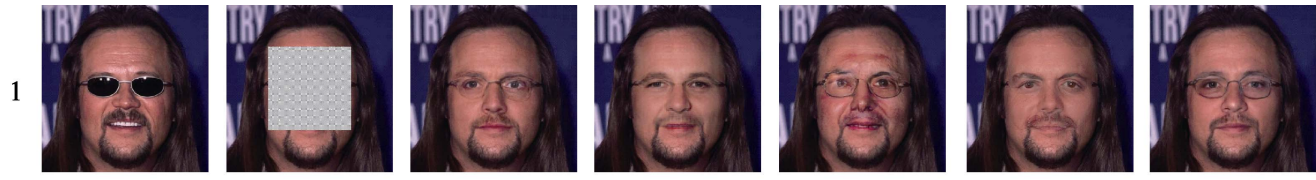

2
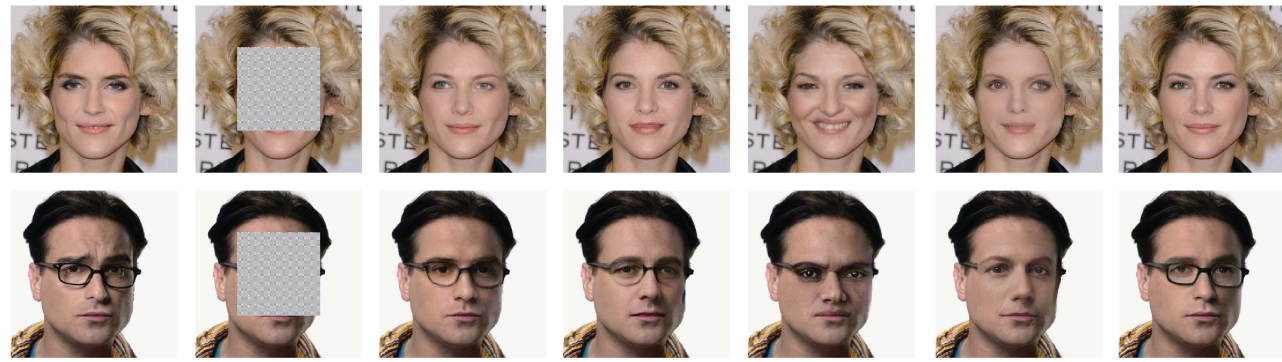

4
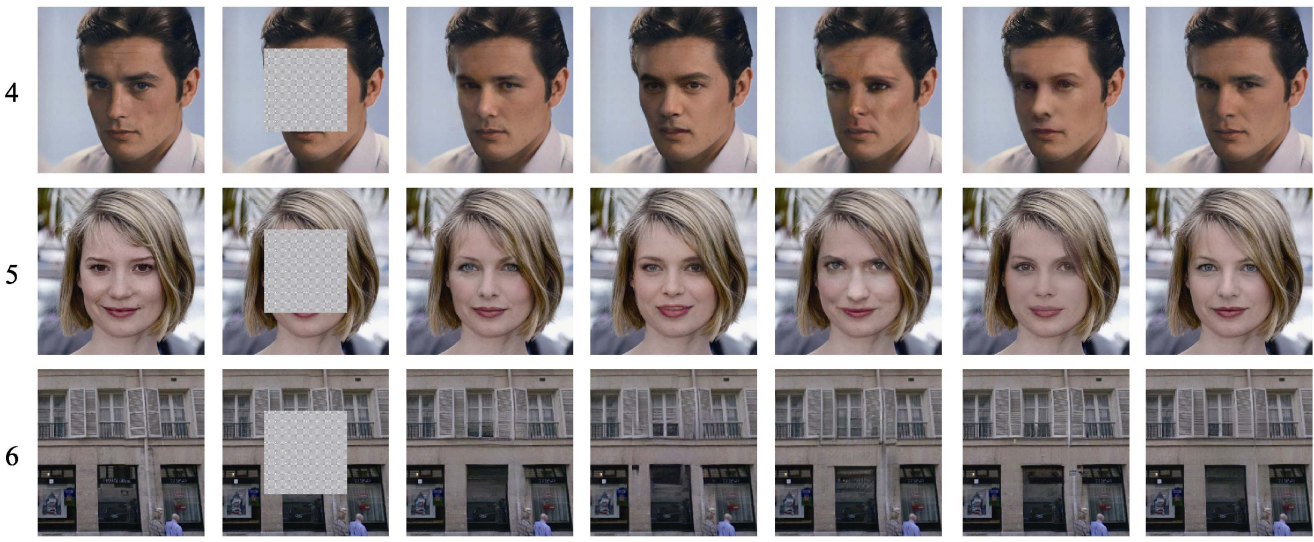

7
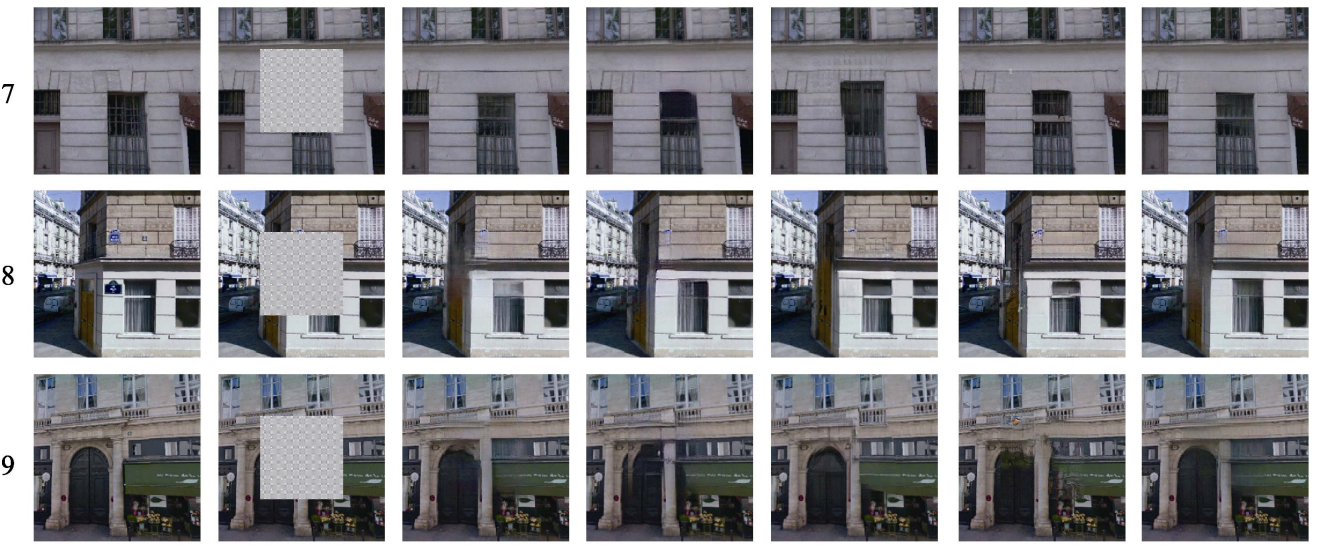

10

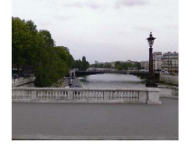

a. 原图

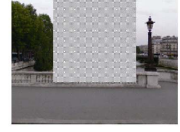

b. 受损图像

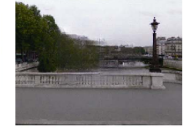

c. Shift-Net ${ }^{[5]}$

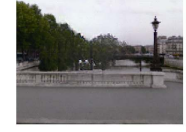

d. GMCNN ${ }^{[28]}$

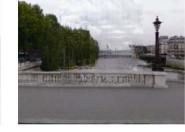

e. $\mathrm{PIC}{ }^{[29]}$

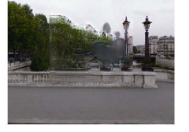

f. PEN-NET ${ }^{[16]}$

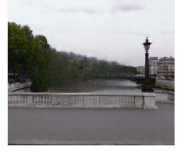

g. 本文

图 5 不同模型的图像修复结果对比

表 1 在 CelebA-HQ 数据集上中心矩形掩码修复对比

\begin{tabular}{lccr}
\hline \multicolumn{1}{c}{ 模型 } & PSNR/dB & SSIM & \multicolumn{1}{c}{ FID } \\
\hline Shift-Net $^{[5]}$ & 26.5069 & 0.8932 & 6.9474 \\
GMCNN $^{[28]}$ & 26.1833 & 0.8928 & 7.6649 \\
PIC $^{[29]}$ & 24.6115 & 0.8728 & 6.7809 \\
PEN-NET $^{[16]}$ & 25.5024 & 0.8810 & 10.6293 \\
本文 & $\mathbf{2 6 . 7 5 5 2}$ & $\mathbf{0 . 8 9 5} \mathbf{1}$ & $\mathbf{6 . 7 0 9 9}$ \\
\hline
\end{tabular}

注. 粗体表示各指标的最佳值.
表 2 Paris StreetView 数据集上中心矩形掩码修复对比

\begin{tabular}{lccc}
\hline \multicolumn{1}{c}{ 模型 } & PSNR/dB & SSIM & FID \\
\hline Shift-Net $^{[5]}$ & 24.9759 & 0.8425 & 46.2299 \\
GMCNN $^{[28]}$ & 24.1038 & 0.8316 & 47.6039 \\
PIC $^{[29]}$ & 23.7279 & 0.8294 & 45.0505 \\
PEN-NET $^{[16]}$ & 23.5907 & 0.8204 & 63.8251 \\
本文 & $\mathbf{2 5 . 5 3 1 3}$ & $\mathbf{0 . 8 4 9 2}$ & $\mathbf{4 4 . 7 4 6 6}$ \\
\hline
\end{tabular}

注. 粗体表示各指标的最佳值. 


\section{3 用户评价}

为了评价图像修复结果给人的直观感受, 本 文从基于 CelebA-HQ 数据集的测试结果中随机抽 取 50 组原始图像及其修复结果, 每组图像中真假 图像的排序是随机的. 20 名受试者受邀对这 50 组 图像进行观察, 并判断哪幅图像为真实图像. 这 20 名受试者判断的正确率如表 3 所示.

表 3 测试者对图像真假判断的正确率

\begin{tabular}{cc||cc}
\hline 测试者 & 正确率 & 测试者 & 正确率 \\
\hline 1 & 0.22 & 11 & 0.44 \\
2 & 0.30 & 12 & 0.46 \\
3 & 0.30 & 13 & 0.48 \\
4 & 0.30 & 14 & 0.48 \\
5 & 0.36 & 15 & 0.50 \\
6 & 0.36 & 16 & 0.54 \\
7 & 0.36 & 17 & 0.56 \\
8 & 0.36 & 18 & 0.56 \\
9 & 0.36 & 19 & 0.58 \\
10 & 0.42 & 20 & 0.62 \\
\hline
\end{tabular}

表 3 中，受试者对修复的人脸图像的真假判断 的正确率按从小到大进行升序排列. 可以看出, 这 20 名受试者中 1 14 号分辨图像真假的正确率都低 于 0.5 , 由此说明, $70 \%$ 的受试者对于大部分的测试 图像无法分辨出哪幅图像是真实图像，哪幅是合 成的图像. 且表中分辨图像真假的正确率最高也

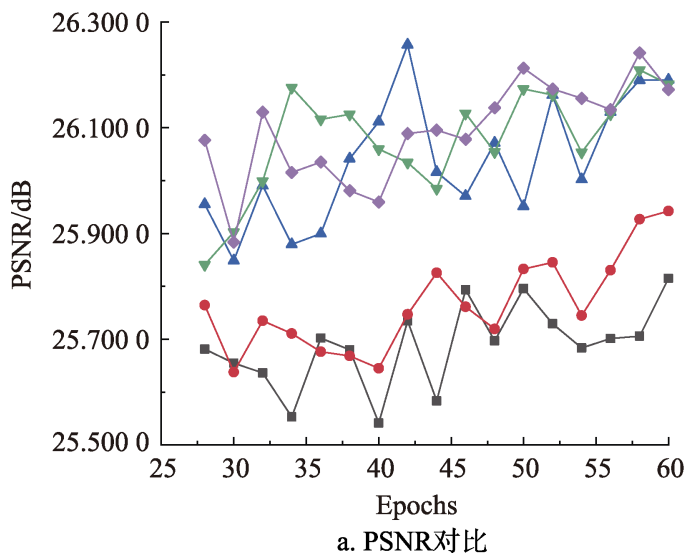

仅为 0.62 , 可见本文模型在修复人脸图像时, 修复 结果十分符合人的直观感受, 甚至比原始图像更 令人感到真实. 这点也符合对图 5 中第 2 行与第 5 行的观察结果.

\section{4 基于 Paris StreetView 数据集的本文模块 消融实验}

为了验证本文提出的模块在图像修复网络中 的作用, 基于 Paris StreetView 数据集对不同模型 进行了消融实验. 采用 Shift-Net作为基础模型, 并 通过在其上添加各类额外的结构作为对照模型. 为了公平比较，所有模型均以相同的设置进行训 练. 测试图像的大小为 $256 \times 256$ 像素, 中心矩形掩 码为 $120 \times 120$ 像素. 图 6 所示为模块消融实验的定 量评价结果. 图 6a 所示为基于 Paris StreetView 数 据集不同模型的 PSNR 对比结果, 可以看出, 采用 Res2U-Net 可以明显地提高修复图像的 PSNR 指标, 而引人 DMSCA 模块对提升修复图像的 PSNR 指标 也具有一定的帮助. 图 $6 \mathrm{~b}$ 所示为基于 Paris StreetView 数据集的各对照模型 FID 对比结果, 其 中, Res2U-Net 显著降低了修复结果的 FID 值. 与 CA 相比, DMSCA 使修复结果可以获得更低的 FID 值. 由此说明, 本文 Res2U-Net 和 DMSCA 模 块在像素层面和感知层面均明显提高了图像修复 的质量.

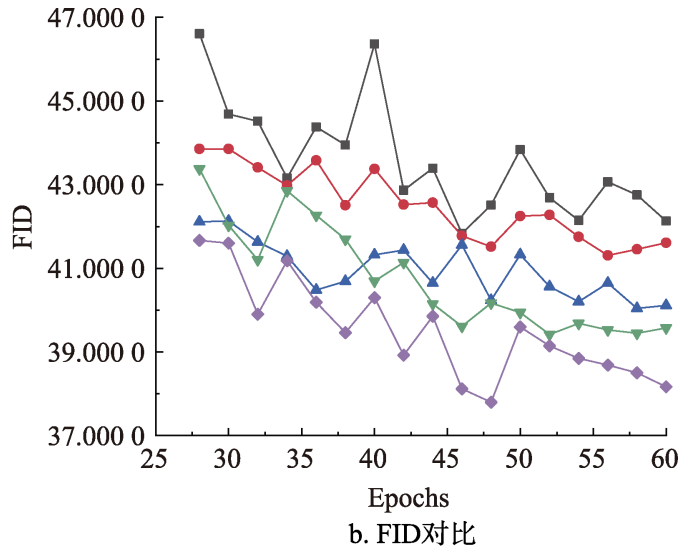

图 6 采用不同模型消融实验定量评价结果

图 7 所示为本文完整模型与各种消融变体模 型对街景图像进行修复的结果定性对比. 从图 7c 与图 7d可以看出, 将 DMSCA 加人基础模型后, 修 复得到的边缘纹理会变得更加清晰; 从图 7c 与图 $7 \mathrm{e}$ 可以看出, 在基础模型中采用 Res2U-Net 结构 后, 修复区域与周围区域之间具有更好的语义结 构连续性, 在视觉观察上更加和谐自然; 从图 $7 \mathrm{f}$
与图 $7 \mathrm{~g}$ 可以看出, 相较于采用 CA 模块的模型, 本 文在基础模型中加人 DMSCA 模块能使修复后的 图像区域具有更为平滑的纹理结构.

\section{5 基于 CelebA-HQ 数据集的损失函数消融 实验}

为了验证各种损失函数在图像修复网络中的 作用, 本节采用 CelebA-HQ 数据集对提出的图像 


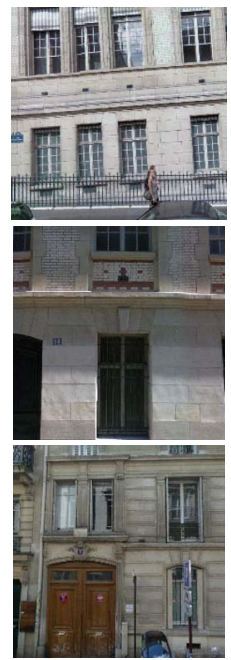

a. 原图

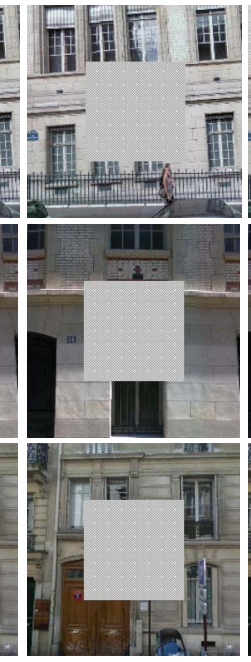

b. 受损图像

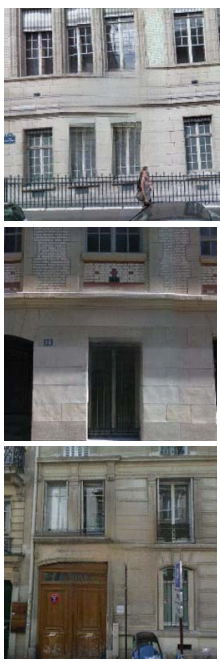

c. 基础模型 ${ }^{[5]}$

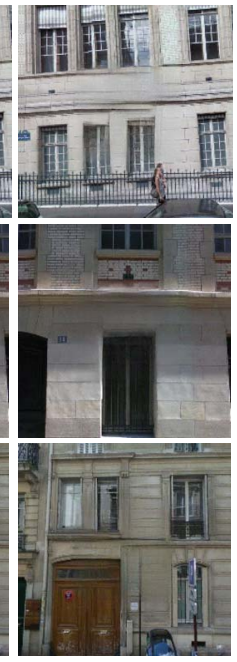

d. DMSCA

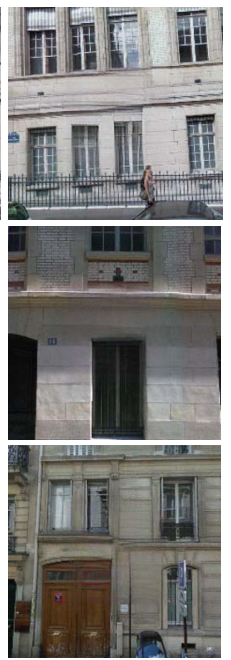

e. Res2U-Net f. 在 e 中添加 CA

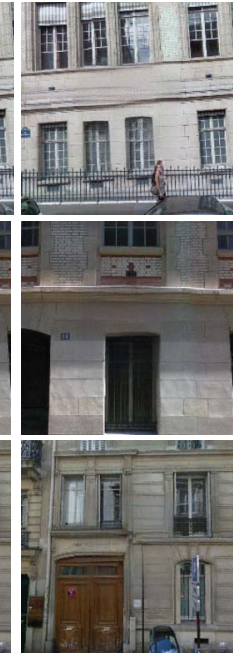

g. 完整模型

图 7 采用不同模型消融实验图像修复结果对比

修复模型进行消融实验. 首先, 基于联合训练损失 对修复网络进行训练, 将训练得到的图像修复模 型作为基础模型; 然后，分别移除其中一个损失函 数进行训练得到 5 个不同的消融模型. 所有模型均 设置相同参数进行训练, 测试图像大小为 $256 \times 256$ 像素, 中心矩形掩码大小为 $128 \times 128$ 像素. 表 4 所 示为损失函数消融实验结果的定量比较. 可以看 出, 当从联合训练损失中去除像素重构损失 $L_{\mathrm{rec}}$ 与 内容损失 $L_{\mathrm{cont}}$ 以后, 图像修复结果的各项评价指 标均出现了一定程度的劣化. 当去除风格损失 $L_{\mathrm{sty}}$ 与总变分损失 $L_{\mathrm{tv}}$ 以后，图像修复结果的 2 个指标 SSIM 和 FID 略有改善, 但 PSNR 指标出现了下降. 当去除对抗损失 $L_{\mathrm{adv}}$ 以后，图像修复结果的 PSNR 和 SSIM 指标明显提高, 但 FID 指标出现了明显的 劣化.

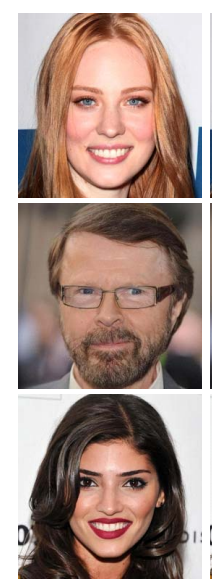

a. 原图

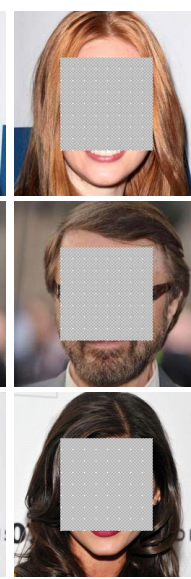

b. 受损图像

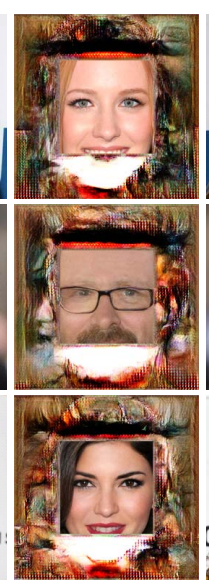

c. 去除 $L_{\text {rec }}$

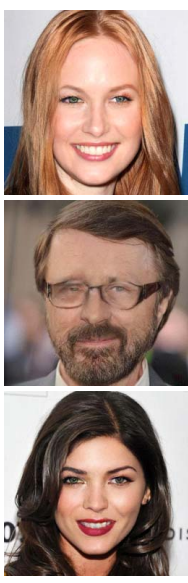

d. 去除 $L_{\text {cont }}$
表 4 在 CelebA-HQ 数据集上损失函数消融实验对比

\begin{tabular}{lrrr}
\hline \multicolumn{1}{c}{ 模型 } & PSNR/dB & SSIM & \multicolumn{1}{c}{ FID } \\
\hline 去除 $L_{\text {rec }}$ & 9.5230 & 0.2032 & 260.3084 \\
去除 $L_{\text {cont }}$ & 26.4163 & 0.8932 & 7.3336 \\
去除 $L_{\text {sty }}$ & 26.7032 & 0.8965 & $\mathbf{6 . 4 6 1 ~ 1}$ \\
去除 $L_{\text {tv }}$ & 26.6977 & 0.8953 & 6.6692 \\
去除 $L_{\text {adv }}$ & $\mathbf{2 7 . 0 4 6 9}$ & $\mathbf{0 . 9 0 2 6}$ & 9.5648 \\
完整模型 & 26.7552 & 0.8951 & 6.7099 \\
\hline
\end{tabular}

注. 粗体表示各指标的最佳值.

图 8 所示为损失函数消融实验结果的定性比 较. 从图 8c 与图 $8 \mathrm{~h}$ 可以看出, 当去除联合训练损 失中的像素重构损失 $L_{\mathrm{rec}}$ 后, 修复模型无法有效地 重构输人图像中的未受损区域. 从图 $8 \mathrm{~d}$ 与图 $8 \mathrm{~h}$ 可 以看出, 当去除联合训练损失中的内容损失 $L_{\mathrm{cont}}$ 后, 图像修复结果出现了内容上的失真, 例如, 第

图 8 损失函数消融实验图像修复结果对比 
2 张图片中的眼镜结构发生了扭曲. 从图 $8 \mathrm{e}$ 与图 $8 \mathrm{~h}$ 可以看出, 当去除联合训练损失中的风格损失 $L_{\mathrm{sty}}$ 后, 修复图像中部分区域在风格上较为不自然 (如图 $8 \mathrm{e}$ 第 3 行中的嘴唇区域). 从图 $8 \mathrm{f}$ 与图 $8 \mathrm{~h}$ 可 以看出, 当去除联合训练损失中的总变分损失 $L_{\mathrm{tv}}$ 后, 局部细节的修复质量出现了下降(如图 8f 第 2 行中的眼部区域). 从图 $8 \mathrm{~g}$ 与图 $8 \mathrm{~h}$ 可以看出, 当去 除联合训练损失中对抗损失 $L_{\mathrm{adv}}$ 后, 修复后的图 像内容出现了纹理模糊 (如图 $8 \mathrm{~g}$ 第 2 行中修复出的 胡须区域).

\section{6 可视化分析实验}

本文提出的 DMSCA 模块旨在利用生成的注 意力图来减少跳跃连接给解码器带来的干扰, 以
使修复模型能更有效地利用编码器中的低级特征. 为了观察和分析 DMSCA 模块生成的注意力图的 作用效果, 本文对编码器网络第 1 层输出的特征和 DMSCA 模块处理后的特征做了可视化处理, 二者 尺度均为 $128 \times 128$ 像素. 图 9 所示为 DMSCA 作用 效果的可视化图. 其中, 图 9c 和图 9d 以热力图来 表示特征数值的大小, 暖色表示特征数值较大, 冷 色表示特征数值较小. 可以看出, 经过 DMSCA 模 块处理以后中央破损区域的颜色从青色变为紫色, 说明受损区域所造成的干扰得到了抑制; 另外，墙 壁纹理以及木板花纹的特征数值得到了提升, 说 明 DMSCA 模块能够自适应增强不同尺度重要特 征的权重.

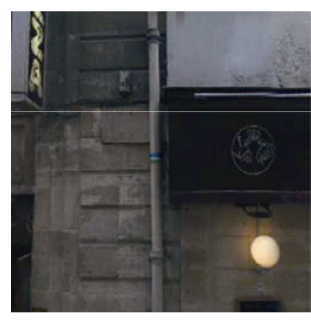

a. 原图

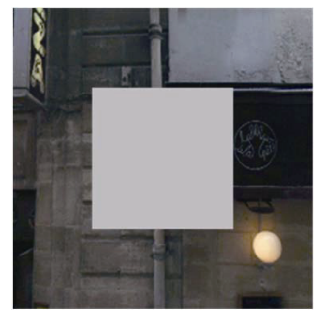

b. 受损图像

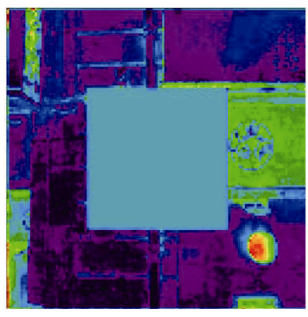

c. 编码器特征

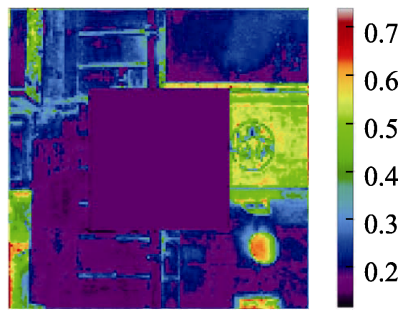

d. DMSCA输出特征

图 9 DMSCA 作用效果可视化

\section{4 结 语}

本文提出了一种基于多尺度 CA 与分层残差 主干网络的图像修复模型. 通过使用分层和残差 机制, 增强生成器对图像中远距离特征间的信息 相关性的捕获能力, 进而提升模型对多尺度特征 提取和表达的能力. 通过将 DMSCA 模块嵌人到跳 跃连接结构中, 使得模型更有效地利用编码器中 的低级特征来提升图像的修复效果. 实验表明, 本 文模型在各种图像修复任务中都能获得具有逼真 细节和合理语义结构的修复结果, 定性与定量评 估均达到先进水平. 实验中设置 Batch Size 为 1 , 是因为修复模型采用了 Shift-Net 中的移位连接层, 该结构的性能会受到 Batch Size 的影响. 经过测算, 模型的显存占用为 $2587 \mathrm{MB}$, 修复一张图片的平均 时间约为 $200 \mathrm{~ms}$, 具有实用意义. 未来可以尝试将 DMSCA 模块和 Res2U-Net 结构应用到诸如超分辨 率和有条件的图像生成等图到图转换的任务中.

\section{参考文献(References):}

[1] Bertalmio M, Sapiro G, Caselles V, et al. Image inpainting[C]
//Proceedings of the 27th Annual Conference on Computer Graphics and Interactive Techniques. New York: ACM Press, 2000: 417-424

[2] Barnes C, Shechtman E, Finkelstein A, et al. PatchMatch: a randomized correspondence algorithm for structural image editing[J]. ACM Transactions on Graphics, 2009, 28(3): Article No.24

[3] Ronneberger O, Fischer P, Brox T. U-Net: convolutional networks for biomedical image segmentation[C] //Proceedings of the Medical Image Computing and Computer-Assisted Intervention. Heidelberg: Springer, 2015: 234-241

[4] Liu G L, Reda F A, Shih K J, et al. Image inpainting for irregular holes using partial convolutions[C] //Proceedings of European Conference on Computer Vision. Heidelberg: Springer, 2018: 89-105

[5] Yan Z Y, Li X M, Li M, et al. Shift-Net: image inpainting via deep feature rearrangement[C] //Proceedings of European Conference on Computer Vision. Heidelberg: Springer, 2018: 3-19

[6] Liu H Y, Jiang B, Xiao Y, et al. Coherent semantic attention for image inpainting[C] //Proceedings of the IEEE International Conference on Computer Vision. Los Alamitos: IEEE Computer Society Press, 2019: 4170-4179

[7] Yu J H, Lin Z, Yang J M, et al. Generative image inpainting with contextual attention[C] //Proceedings of the IEEE Conference on Computer Vision and Pattern Recognition. Los Alamitos: IEEE Computer Society Press, 2018: 5505-5514

[8] Ren Y R, Yu X M, Zhang R N, et al. StructureFlow: image inpainting via structure-aware appearance flow[C] //Proceed- 
ings of the IEEE International Conference on Computer Vision. Los Alamitos: IEEE Computer Society Press, 2019: 181-190

[9] Pathak D, Krähenbühl P, Donahue J, et al. Context encoders: feature learning by inpainting[C] //Proceedings of the IEEE Conference on Computer Vision and Pattern Recognition. Los Alamitos: IEEE Computer Society Press, 2016: 2536-2544

[10] Iizuka S, Simo-Serra E, Ishikawa H. Globally and locally consistent image completion[J]. ACM Transactions on Graphics, 2017, 36(4): Article No.107

[11] Yu J H, Lin Z, Yang J M, et al. Free-form image inpainting with gated convolution[C] //Proceedings of the IEEE International Conference on Computer Vision. Los Alamitos: IEEE Computer Society Press, 2019: 4471-4480

[12] Nazeri K, Ng E, Joseph T, et al. EdgeConnect: generative image inpainting with adversarial edge learning[OL] [2020-07-24]. https://arxiv.org/abs/1901.00212

[13] Jaderberg M, Simonyan K, Zisserman A, et al. Spatial transformer networks[C] //Proceedings of the Advances in Neural Information Processing Systems. Cambridge: MIT Press, 2015: 2017-2025

[14] Zhang Y L, Li K P, Li K, et al. Image super-resolution using very deep residual channel attention networks[C] //Proceedings of European Conference on Computer Vision. Heidelberg: Springer, 2018: 294-310

[15] Wang N, Li J Y, Zhang L F, et al. MUSICAL: multi-scale image contextual attention learning for inpainting[C] //Proceedings of the 28th International Joint Conference on Artificial Intelligence. San Francisco: Morgan Kaufmann, 2019: 3748-3754

[16] Zeng Y H, Fu J L, Chao H Y, et al. Learning pyramid-context encoder network for high-quality image inpainting[C] //Proceedings of the IEEE Conference on Computer Vision and Pattern Recognition. Los Alamitos: IEEE Computer Society Press, 2019: 1486-1494

[17] Xie C H, Liu S H, Li C, et al. Image inpainting with learnable bidirectional attention maps[C] //Proceedings of the IEEE International Conference on Computer Vision. Los Alamitos: IEEE Computer Society Press, 2019: 8858-8867

[18] Fang Y C, Li Y F, Tu X K, et al. Face completion with hybrid dilated convolution[J]. Signal Processing: Image Communication, 2020, 80: 115664
[19] Isola P, Zhu J Y, Zhou T H, et al. Image-to-image translation with conditional adversarial networks[C] //Proceedings of the IEEE Conference on Computer Vision and Pattern Recognition. Los Alamitos: IEEE Computer Society Press, 2017: 1125-1134

[20] Gao S H, Cheng M M, Zhao K, et al. Res2Net: a new multi-scale backbone architecture[J]. IEEE Transactions on Pattern Analysis and Machine Intelligence, 2019, 43(2): 652-662

[21] Hu J, Shen L, Sun G. Squeeze-and-excitation networks[C] //Proceedings of the IEEE Conference on Computer Vision and Pattern Recognition. Los Alamitos: IEEE Computer Society Press, 2018: 7132-7141

[22] Ioffe S, Szegedy C. Batch normalization: accelerating deep network training by reducing internal covariate shift[C] //Proceedings of the 32nd International Conference on Machine Learning. Lille: PMLR Press, 2015: 448-456

[23] Johnson J, Alahi A, Li F F. Perceptual losses for real-time style transfer and super-resolution[C] //Proceedings of European Conference on Computer Vision. Heidelberg: Springer, 2016: 694-711

[24] Goodfellow I J, Pouget-Abadie J, Mirza M, et al. Generative adversarial nets[C] //Proceedings of the 27th International Conference on Neural Information Processing Systems. Cambridge: MIT Press, 2014: 2672-2680

[25] Simonyan K, Zisserman A. Very deep convolutional networks for large-scale image recognition[OL]. [2020-07-24]. https://arxiv.org/abs/1409.1556

[26] Karras T, Aila T, Laine S, et al. Progressive growing of GANs for improved quality, stability, and variation[OL]. [2020-07-24]. https://arxiv.org/abs/1710.10196

[27] Doersch C, Singh S, Gupta A, et al. What makes Paris look like Paris?[J]. ACM Transactions on Graphics, 2012, 31(4): Article No.101

[28] Wang Y, Tao X, Qi X J, et al. Image inpainting via generative multi-column convolutional neural networks[C] //Proceedings of the Advances in Neural Information Processing Systems. Cambridge: MIT Press, 2018: 329-338

[29] Zheng C X, Cham T J, Cai J F. Pluralistic image completion[C] //Proceedings of the IEEE Conference on Computer Vision and Pattern Recognition. Los Alamitos: IEEE Computer Society Press, 2019: 1438-1447 